\title{
Is the many body localization transition accessible to computation?
}

\section{Quantum chaos challenges many-body localization} Authors: J. Šuntajs, J. Bonča, T. Prosen, and L. Vidmar arXiv:1905.06345

2. Distinguishing localization from chaos: challenges in finite-size systems Authors: D. A. Abanin, J. H. Bardarson, G. De Tomasi, S. Gopalakrishnan, V. Khemani, S. A. Parameswaran, F. Pollmann, A. C. Potter, M. Serbyn, and R. Vasseur arXiv:1911.04501

\section{Can we study the many-body localisation transition?}

Authors: Rajat K. Panda, Antonello Scardicchio, Maximilian Schulz, Scott R. Taylor, and Marko Žnidarič

arXiv:1911.07882

\section{Recommended with a Commentary by Ehud Altman, UC Berkeley}

The wide interest in the many-body localization (MBL) phase transition stems, to a large extent, from the fact that it presents a sharp boundary between quantum and classical behavior of macroscopic matter. While in a thermalizing state quantum correlations are rapidly washed out by the dynamics, giving way to classical hydrodynamics at long times, in the MBL state such correlations can persist indefinitely and crucially contribute to the dynamics. The papers I recommend highlight the unconventional nature of this transition and offer some insight as to why it has so far resisted a reliable characterization using numerical simulation or experiments. Paper (1) above attempts to challenge our current understanding of the many-body localization transition. Papers (2) and (3) respond to this challenge and, in doing so, highlight potential obstructions to observing the transition using conventional numerical approaches and possibly also near term experiments.

In the last decade there has been significant progress in understanding many-body localization. There are good theoretical arguments suggesting that the stability of the localized phase is ensured, in a sufficiently disordered one-dimensional system, due to vanishing probability for formation of a resonant cluster that spans the entire system. Within this picture, the transition to a thermalizing phase occurs at a finite disorder strength, through an avalanche process triggered by an extremely dilute set of microscopic sites. Thus, the critical point exhibits insulating-like behavior with thermalization times that are exponentially long with the system length $L$. On approaching the transition from the thermal side, 
the system appears insulating on short length scales, below the diverging correlation length $\xi$. At longer scales the system is expected to show sub-diffusive transport: a system of length $L$ thermalizes at a time $\tau_{t h} \sim L^{z}$, with $z>2$. Moreover, the dynamical exponent $z$ changes continuously and diverges at the MBL critical point, leading to extreme slowing down of the dynamics even before the transition has been reached. The phase transition in the dynamics is also accompanied by a fundamental change of the many-body spectrum. In the localized phase the spectrum is expected to show Poisson statistics, while in the thermalizing phase the energy levels should show strong repulsive correlations at energy differences smaller than the Thouless energy, or inverse thermalization time $\left(E_{T}=\hbar / \tau_{t h}\right)$.

Paper (1) presents a challenge to this picture through analysis of the many-body energy level correlations. First, from the spectral form factor (SFF), a generating function for the energy level correlations, the authors extract a global thermalization time (inverse of the Thouless energy). The thermalization time can be reliably extracted in this scheme for moderate disorder $W$ deep in the thermal phase, where the authors fit it to the scaling form $\tau_{t h} \sim e^{W / W_{0}} L^{2}$. If this scaling persisted to strong disorder, it would imply that the thermalization time reaches a scale exponentially long with $L$, as it must be in the MBL phase, only at a critical disorder strength $W_{c} \sim L$. In other words, the localization transition would occur only at extensive, rather than finite disorder strength. It is worth noting, that the fitted scaling form is diffusive $(z=2)$, which is probably consistent with the fact that the analysis can only be carried out deep in the thermal phase. It would still be interesting to see if the data is consistent with an alternative scaling ansatz in which the dynamical exponent $z$ increases continuously with $W$. Either way, paper (1) presents another piece of evidence for extensive $W_{c}$ using a more conventional diagnostic of localization: the ratio of adjacent level spacings $r . r$ is known to take one value for Poisson levels and a different value in case of Wigner-Dyson statistics. Calculating $r$ for different disorder strengths and system sizes, the authors show that a good crossing point, indicating a localization transition, is obtained only if $r$ is plotted against the disorder strength scaled as $W / L$.

Do these numerical results challenge the existence of MBL? The authors of paper (2) argue they do not. They caution against extrapolating the scaling behavior of the thermalization time, as seen deep in the thermal phase, all the way to the transition and point to adverse finite size effects that plague numerical studies of localization transitions more generally. Perhaps the most striking analysis they present is a comparison between the results of paper (1) and those they obtain for the model of a single particle on the random regular graph (RRG). The existence of a localization transition had been rigorously established in the latter, yet analysis of the $r$ ratio in systems of moderate size leads to exactly the behavior found in paper (1): a crossing point at a fixed value of $W / L$. Thus with a naive analysis of the RRG model one would also deduce absence of Anderson localization. The question remains though, what is the origin of the strong finite size effects plaguing the many-body localization transition. One intriguing result of paper (3) may offer a clue.

Part of paper (3) is devoted to a careful study of the global and local relaxation time of a system initialized with a domain wall in the spin profile. The local relaxation time $t_{1}$ is defined as the time to transfer a single spin across the domain wall and a global relaxation time $t_{N}$ is the time for transferring $N \sim O(L)$ spins. The authors of paper (3) find $t_{1} \approx e^{W / \tilde{W}}$ and $t_{N} \approx t_{1}(W) N^{z}$. There are two important points to note. First is 
that a sub-diffusive scaling is observed with dynamical exponent $z>2$ and clearly growing with disorder strength in accordance with current understanding of the transition. However, the crucial second observation is that in the accessible small system sizes the relaxation time reaches the Heisenberg time $t_{H} \sim 2^{L} /(W L)$, set by inverse level spacing because of the rapid growth of the local relaxation time, well before the dynamical exponent $z$ had a chance to diverge. The conclusion of this paper is that current exact diagonalization studies are observing a spurious crossover from Wigner-Dyson to Poisson statistics driven by slowing down of the local dynamics. They estimate that the true MBL transition, driven by the global slowing down due to diverging dynamical exponent, would be observable only for system sizes of order $L \gtrsim 50$.

The recommended studies point to the difficulties associated with studying the manybody localization transition using conventional numerical approaches limited to small sizes or experiments limited by time. A natural way to detect the sub-diffusive behavior in experiment is to impose some non-equilibrium profile, such as a density wave, and fit the relaxation of the wave amplitude to a power law $\sim\left(t / t_{1}\right)^{-1 / z}$. Such a fit becomes unreliable for $z \gtrsim 5$ when the experiment is limited to reasonable timescales, due to inevitable decay processes or coupling to the environment making difficult to approach the phase transition at which $z$ diverges. Paper (3) adds another layer of difficulty by pointing to the rapidly growing local timescale $t_{1} \approx e^{W / \tilde{W}}$. To make progress in addressing the MBL transition with controlled numerical calculations it would be important to understand this growth of the local time scales. It stands to reason that this is an essential consequence of the asymmetry between thermalization and localization. Very few thermalizing seeds can destabilize the localized phase, yet a few localized islands are not effective in localizing the bulk. For this reason the true MBL transition occurs at rather large disorder strength that is sufficient to slow the local dynamics appreciably. It remains to be seen if this effect can be mitigated in specially constructed models of MBL. 